\title{
THE ASSOCIATION OF CREATIVITY WITH DIVERGENT AND CONVERGENT THINKING
}

\author{
Syed Fahad Javaid ${ }^{1} \&$ James Paul Pandarakalam ${ }^{2}$ \\ ${ }^{1}$ Department of Psychiatry and Behavioural Sciences, College of Medicine and Health Sciences, \\ United Arab Emirates University, Al Ain, United Arab Emirates \\ ${ }^{2}$ Northwest Boroughs Healthcare NHS Foundation Trust, Hollins Park Hospital \& Alternative Futures \\ Rehabilitation Hospitals, Warrington, United Kingdom
}

received: 2.1.2021;

revised: 14.4.2021;

accepted: 6.5.2021

\section{SUMMARY}

The inspirational aspect of creativity remains shrouded in mystery. Methodological problems have hindered research into creativity, and such a situation makes the interpretation and comparison of studies problematic. The link between creativity and psychopathology is overstated by the print, electronic, and celluloid media. This paper attempts to explain the creative process from a psychological and psychiatric perspective leaving room for different unexplained aspects of generativity for open discussion. A selective survey of the literature was performed to identify scholarly views of creativity and psychopathology. Data sources included PubMed, Google Scholar, and Scopus. The concept of inspiration was examined from psychological, psychopathological, and biological standpoints.

A better understanding of creativity has clinical implications. Psychopathology can facilitate creativity, but it is not the maker of creativity that involves a harmonious blending of divergent and convergent thinking. The present trend in psychiatry of medicalizing all unusual behavior is not at all productive in fostering creativity among children. The cognitively gifted children differ widely from children with autistic spectrum disorders; the creative thinking of gifted children is polythetic, whereas such potentials of autistic individuals are generally monothetic.

The study of creativity helps develop an expanded model of the mind. However, research into creativity has produced contradictory results. The assumed link between creativity and mental disorder could be clarified only when we elucidate the creative process. Further research is needed in regard to the psycho-biological nature of creativity, including genetic links, implications for neuropharmacology, and the treatment of pathology or psychological disorders.

Key words: creativity - divergent thinking - convergent thinking - psychopathology - inspiration

$$
* * * * * *
$$

\section{INTRODUCTION}

Creativity is an ambiguous term with multiple competing definitions and conceptualisations (Grohman et al. 2017). Creativity is a multilevel process with a neurobiological foundation. The current genetic and psychopathological views of creativity are rooted in the reductionist model of consciousness. Without adequate intellectual tools and an expanded model of the brain-mindconsciousness complex, studies of creativity are inevitably erroneous. Within the context of psychology, creativity is generally considered a mental process of expression or generation of a new concept or idea (SaidMetwaly et al. 2017). Some scholars conceptualise creativity as a dichotomous interaction between divergent and convergent thinking (Zhu et al. 2019). Divergent thinking refers to the achievement of a resolution to some problem in which multiple potential solutions are presented and the correct, or most optimal, one is selected (Colzato et al. 2017). Conversely, convergent thinking refers to applying rules or procedures to achieve a single correct solution to some problem (Colzato et al. 2017).

The purpose of this paper is to present different interpretations of creativity with an emphasis on convergent versus divergent perspectives on this issue. We argue that creativity is not necessarily a dichotomous and mutually exclusive relationship between convergent and divergent thinking but, rather, a harmonious blend of these two forms of cognition. In arguing this point, psychological, psychopathological, and biological views of creativity are analysed separately. Based on consideration of each of these two forms of thinking, conclusions are then drawn regarding this paper's thesis.

\section{DIVERGENT AND CONVERGENT THINKING}

Openness and extraversion are considered as characteristics of divergent thinkers (McCrae 1987), but they can be easily misunderstood as introverts with poor social skills and stubborn individuals when others cannot confirm with their views. Divergent thinkers can look eccentric in an intolerant social environment. Other Personality traits of individuals capable of divergent thinking include curiosity, high imagination, momentary gullibility, nonconformity, persistence, ability to tolerate ambivalence, and risk-taking behavior (McCrae 1987).

If divergent thinking is associated with creativity, convergent thinking is linked with typical intelligence. Divergent thinking uses imagination, and convergent 
thinking employs logic; the former is also called creative or horizontal thinking, whereas the latter is labelled as critical or vertical thinking. Genuine creativity involves both types of thinking. In divergent thinking, multiple possible solutions are generated and evaluated. This process is spontaneous and happens typically in a brief span of time in a free-flowing and non-linear fashion. In a healthy person, it is succeeded by convergent thinking, which involves organising information and ideas in a structured manner. In divergent thinking, a single idea may mushroom into multiple complex ideas with unanticipated associations. A single question generates multiple novel answers.

It is convergent thinking that converts divergent thinking into practicality. Students in the schools are trained for convergent thinking. Answering multiplechoice questions (MCQs) require convergent thinking, whereas writing an essay needs divergent thinking. So, students who are just intelligent but have not practiced divergent thinking perform better in examinations with multiple-choice questions. Convergent thinking crystallizes the imaginary product of divergent thinking, and it is an essential part of the entire creative process. Unlike divergent thinking, everybody is capable of convergent thinking, which may get impaired due to psychopathology.

It has been demonstrated that divergent thinking brings about mood swings and not the other way (Chermahini \& Hommel 2012). Studies show that divergent thinking triggers a negative mood, and focused thinking results in a positive mood. Divergent thinkers have high anxiety scores and struggle to tolerate sleep loss when compared to convergent thinkers. An oversleeping child may be thus having divergent thinking patterns. Standard IQ tests can measure convergent thinking, but not necessarily divergent thinking. Electroencephalogram patterns of both types of thinkers have been different (Razoumnikova 2000). While divergent thinking causes amplitude reductions in the cortex's caudal regions in the Theta 1 and 2 bands, convergent thinking causes coherence boosts in the Theta 1 band that is more rightsided and caudal. The considerable soaring of coherence and amplitude signifies a close iteration between both brain hemispheres. Divergent and convergent thinking occurs and blends harmoniously in healthy people. If divergent thinking leads to funnel thinking, convergent thinking is synonymous with tunnel vision.

\section{PSYCHOLOGICAL VIEWS}

Psychologists have been concerned with the distinction between convergent and divergent thinking within a range of domains and contexts (Cheung et al. 2016). One example is creativity, or the ability to generate novel ideas or possibilities that may be used to solve problems, aid in communication, or lead to forms of expression and entertainment. From the perspective of divergent thinking, creativity is viewed as the ability to generate a range of appropriate responses to some task or problem, in which the ideal outcome is not possible to ascertain based on information made available solely within the question. Conversely, a convergent perspective regarding creativity may seem counterintuitive from a psychological standpoint (Wronska et al. 2019). Because convergent thinking involves identifying one optimal solution to a problem, the concept of creativity could be considered paradoxical (Zhu et al. 2019). However, the psychological perspective regarding creativity and convergent thinking is based on how the single correct answer is obtained and the process by which the thinker reaches conclusions related to a particular problem (Eysenck 2003).

Because of these opposing viewpoints, researchers have entertained the possibility that creativity is an amicable fusion of divergent and convergent thinking. Duan and colleagues argued that convergent and divergent thinking share the same cognitive space, with both forms of cognition potentially functioning simultaneously, albeit to different degrees (Duan et al. 2020). It is at this intersection that psychologists would argue that creativity resides. Solving problems according to a set of rules or adhering to logic can still require creativity in terms of the process by which the problem is solved (Yang et al. 2019). When the thinker begins to both utilise rules and procedures and also rebel against these rules or laws, some psychologists would define this as the perfect juxtaposition and that point at which creativity is born (Wronska et al. 2019).

Recently, researchers have examined the psychological underpinnings of convergent versus divergent creativity (de Vries \& Lubart 2019). The study highlights issues about measuring and operationalising the construct of creativity. Limitations exist in the construct validity of tests like the Remote Associates and Alternative Use tasks that are typically used to assess links between creativity and divergent versus convergent thinking (de Vries \& Lubart 2019, Chermahini \& Hommel 2010). Interestingly, these tests are used to measure distinctions between divergent and convergent reasoning but actually involve simultaneous measurement of both forms of cognition (Donnegan et al. 2018, Chermahini \& Hommel 2012). Therefore, research is needed to overcome these problems with construct validity in psychometric assessments of cognition to determine the combined or independent contributions to creativity.

\section{PSYCHOPATHOLOGICAL VIEWS}

Studies have examined the psychopathological links with creativity, divergent thinking, and convergent thinking. They have explored how divergent and convergent thinking reflect mood swings typical of what may be expected in the case of an individual with bipolar or borderline disorder (de Vries \& Lubart 2019, Lu et al. 2017, Jauk et al. 2012). Drawing on a narrative synthesis of the literature, there exists a general agreement that cognitive processes simultaneously affect mood and emotions. Specifically, preparation for a creative task 
that required divergent thinking typically leads to a more positive shift in mood. These findings illustrate the links between mood and thought processes and illustrate how a shift in the mood regarding a creative thinking task is specific to the process involved (Lu et al. 2017, Jauk et al. 2012). Findings from this research also illustrate the psychopathological implications for creativity and convergent versus divergent thinking (Cortes et al. 2019, Japardi et al. 2018).

More recently, researchers have been interested in determining links between creativity and risk-taking using convergent versus divergent thinking tasks. An experimental investigation by Shen and colleagues showed that risk-taking was significantly and inversely correlated with convergent thinking. These findings suggest that creativity may be significantly linked to potentially pathological behaviours and patterns of cognition, such as risk-taking and risky behaviours (Shen et al. 2018). These findings may have implications for links between creativity and sensation seeking, substance abuse and other issues related to pathology, and the identification of potential causes of psychological disorders (Duan et al. 2020, Shen et al. 2018).

The neurobiological model of schizophrenia suggests that a deficit in information-processing systems could contribute to this disorder's symptomatology (Braff \& Greyer 1990, Combalt \& Kellp 1994).

Seminal research from Carson and colleagues offers relevance in understanding creativity and psychopathology, including with respect to the phenomenon of latent inhibition (Carson et al. 2003). Latent inhibition is an attentional phenomenon in which repeated pre-exposure to a stimulus that is not reinforced retards future associability to that stimulus. Correlation between latent inhibition and openness among high-achieving individuals is considerable and highly substantial, and reduced latent inhibition has been associated with psychopathological conditions (Carson et al. 2003). Carson has opined that creative people have displayed elevated risk for certain forms of psychopathology, including mood disorders, schizophrenia spectrum disorders, and alcoholism, and has suggested a model of shared vulnerability between creativity and psychopathology (Carson 2011, 2014). Specifically, Carson argued that "the biological determinants conferring risk for psychopathology interact with protective cognitive factors to enhance creative ideation" (Carson 2011).

\section{SCHIZOPHRENIA AND CREATIVITY}

If the views linking psychopathology with creativity are accepted, creativity and schizophrenia will become only a "neurological difference." Andreasen questioned the hypothesis regarding a connection between creativity and schizophrenia, based on the recognition that schizophrenia generates unusual perceptions leading to creativity (Andreasen 1987). She argued that creative thinking and psychotic thought process are not identical but are analogous in some respects. A creative person experiences an attention surplus, whereas a person with schizophrenia suffers from an attention deficit.

Overinclusion refers to a widening of the boundaries of concepts resulting in the grouping together of disconnected thoughts. Individuals with schizophrenia tend to make unusual associations, resulting in over-inclusive thinking, due to a failure in the usual filtering of stimuli by dysfunctional gating systems (Preti 1995). Higher cognitive individuals also show a "pseudo over-inclusive thinking" because of their capacity to conceive and utilize two or more contradictory concepts simultaneously and undisturbed (Rothenberg 1971). Bleuler has described intellectual ambivalence as a characteristic of schizophrenia, and it is only superficially similar to the janusian process of creative cognition or oppositional thinking, which involves conceiving two or more opposites or antitheses simultaneously (Bleuler 1950).

The janusian thinking process has been assessed in controlled experiments using the Kent-Rosanoff word association test (O'Brian \& Weingartner 1970, Schwartz 1978). In contrast to the creative thinker who is fully aware of logical contradictions, a person with schizophrenia is not conscious of his utterances' contradictory nature. When Albert Einstein made the formulation leading to the general theory of relativity that a man falling from the roof of a house was both in motion and at rest simultaneously, he was fully aware of the contradictory nature of this janusian thinking and was not in a schizophrenic mode of cognition (Rothenberg 1979). Another example is Frank Lloyd Wright's revolutionary design of Fallingwater, in which nature and interior space coexist. It was in highly creative writers, visual artists, and scientists, the janusian process was initially identified (Rothenberg 1973, 1982). Fluency of associations observed among creative individuals is mistaken for over-inclusive thinking (Gathercole 1965). Creative individuals may draw advantage from higher levels of associative thinking, as their brains can effectively process increased sensory input without cognitive overload.

Research also illustrates the association between creativity and 'schizotypy,' or a theory that symptoms of schizophrenia exist on a continuum based on their severity and level of dysfunction (McDonald et al. 2021, Rominger et al. 2017). According to this theory, some symptoms of schizophrenia are adaptative and socially advantageous, with creativity being an example (Rominger et al. 2017)

McDonald and colleagues collected psychometric data on the relationship between positive schizotypy and creative experience. The participants completed an online battery of instruments to assess schizotypy and creative experience. Results demonstrated that suspiciousness buffered the relationship between unusual perceptual experiences and magical thinking. Creative experiences were significantly related to positive affect and power/pleasure, although no other measures of creativity were significantly associated with schizotypy (McDonald et al. 2021). These findings appear to suggest 
that suspiciousness is a symptom of schizophrenia that may also be presented in individuals who report higher than average magical thinking and offer some weak correlational evidence of the relationship between positive schizotypy and one dimension of creativity.

Earlier research using neuroimaging evidence has shown that allusive thinking, in which remote associations are exhibited, and top-down auditory inhibition skills both predict positive schizotypy and creativity (Rominger et al. 2017). Rominger and colleagues sampled individuals who displayed a varying degree of positive schizotypy, seeking to assess auditory inhibition and creative thinking. The Dichotic Listening Test demonstrated that creative thinking and positive schizotypy were significantly related, and both of these traits were associated with common relationships. Creative thinking was more strongly linked with positive schizotypy when there was lower inhibitory control present in the auditory test (Rominger et al. 2017).

\section{BIPOLAR MOOD DISORDER AND CREATIVITY}

Contrary to popular belief in cognitive sciences linking creativity and schizophrenia, the creative writers resemble more in cognitive and conceptual style to those experiencing a bipolar disorder than people with schizophrenia (Andreasen \& Powers 1975). The overinclusiveness of persons with mania tends to be based on bizarre associations, whereas those of the writers are due to imaginative recognition of original and fresh associations. The creative writers can control flights of fantasy, but the individuals with mania suffer from destructive racing of thoughts. The "Bing Bang" of creativity culminates rapidly into a "Big Crunch" of destruction. Albert Einstein, who claimed that he used to discard new ideas every two minutes, is an excellent example to distinguish the productive and discerning racing of thoughts of genuine creativity from the racing of thoughts in individuals with mania. Schuldberg studied the overlap between schizotypal and hypomanic traits and generativity and suggested that affective symptoms may be more important than primary process thinking in determining creativity within a general Population (Schuldberg 1990). The fluctuation of thoughts experienced by the creative individual can sometimes be mistaken for mood swings.

Greenwood offered conceptual and theoretical insight into the relationship between these mood swings in creative people and those with bipolar disorder, suggesting that traits of creativity have been increasingly placed within the spectrum of bipolar disorder (Greenwood 2020). According to Greenwood, an improved conceptualization of creativity and bipolar would allow for high doses of risk variants to be associated with psychopathology, but mild to moderate being associated with advantageous traits. The reconceptualization of creativity may better serve the popu- lation regarding reducing stigmatisation and improving the alignment of psychotherapeutic interventions with the severity and type of symptoms. This view of bipolar disorder and its symptoms as expressions of creativity would lead to improved explanations of population variation in personality and temperament, as well as the distribution of creativity (Greenwood 2020). The links between bipolar disorder and creativity suggest that the latter is associated with an alternating state of mind and mood. These fluctuations in thought and mood may spark creativity and lead to sudden manifestations of creative expression (Pandarakalam 2005).

Rybakowski and Klonowska have made an experimental assessment of the elements of creativity and schizotypy features in individuals with bipolar disorder, compared with healthy control subjects to investigate the effect of depression or mania and the features of schizotypy on the measurements of creativity (Rybakowski \& Klonowska 2011). Creativity was assessed using the Revised Art Scale and the inventiveness dimension of the Berlin Intelligence Structure Test. In their study, the individuals with bipolar disorder obtained significantly higher scores on all dimensions of schizotypy and creativity scales compared with the controls. It is likely that the relationship between bipolar disorder and creativity is mediated by a wide range of factors that have not been fully considered in the literature. These factors limit the ability to which the links between creativity, bipolar disorder, and positive schizotypy can be understood.

\section{BIOLOGICAL VIEWS}

Psycho-biological researchers have been able to measure physiological systems that function in response to various forms of thinking to understand this issue better. Chermahini and Hommel were among the first to identify the links between neurochemical activity with creativity and discover implications for convergent and divergent thinking (Chermahini \& Hommel 2010). They measured the association between eye blink rates and dopamine production when engaged in divergent and convergent thinking. Drawing on a sample of 117 adults, they reported significant correlations between thinking paradigms and both spontaneous eye blink rate and dopaminergic functioning (Chermahini \& Hommel 2010). They also argue that eye blink rates significantly predict flexibility concerning divergent and convergent thinking, though in different forms. Medium levels of eye blinking were found to be linked to the highest level of cognitive flexibility. When engaged in convergent thinking, participants demonstrated significantly stronger positive correlations between eye blink rates/dopaminergic function and intelligence (Chermahini \& Hommel 2010). These findings appear to suggest that higher levels of dopamine inhibit convergent thinking processes. 
Sultan Tarlaci has opined that it may be dopamine excess or dopamine reserve and not dopamine overactivity that may characterise creativity (Tarlaci 2014). Such a distinction is important to spare creativity from being interpreted as the hyperactivity of dopamine in the schizophrenia process.

Jauk and colleagues examined electroencephalogram alpha activity when human subjects were engaged in divergent and convergent cognitive processing tasks in order to understand creativity. Results showed that responses given in divergent thinking tasks were more original than in convergent thinking. Furthermore, divergent thinking was associated with more taskrelated electrical alpha power than convergent thinking in both word association and alternate use tasks (Jauk et al. 2012). These findings illustrate the psychobiological roots of creative thinking and appear to suggest that synchronisation of electrical alpha activity underpins more creative and divergent thinking (Jauk et al. 2012, Japardi et al. 2018, Shen et al. 2018). This enhanced synchronisation may be related to the parietal and frontal cortices' distinct involvement in individuals who have lower versus higher levels of creativity.

Japardi and colleagues used functional magnetic resonance imaging to assess convergent and divergent thinking implications for creative problem solving (Japardi et al. 2018). The study assessed brain responses in groups of participants who were considered to be exceptionally functioning versus those who were considered unexceptional. Results showed that task performance did not differ significantly between the two groups, despite differences in regard to functional magnetic resonance imaging activity. These findings illustrate that individuals who are perceived to be of exceptional levels of creativity do not differ in performance from those who are not considered to be of exceptional levels of creativity, despite their different brain activity patterns, particularly within divergent thinking tasks (Mekern et al. 2019, Donnegan et al. 2018).

\section{THE ENIGMA OF INSPIRATION}

Creativity is regarded as the product of inspiration or creative imagination combined with meticulous, disciplined effort. The hypothesis of interactive creativity explains the Edisonian perception of invention as $1 \%$ inspiration and $99 \%$ perspiration; it assumes that the inspirational aspect has a mysterious unconscious component. The rapid technological growth of recent times has taken place without any noticeable changes in the human brain's development. Excavation at the archaeological site of "Danger Cave" near the Great Salt Lake in Utah shows a record of human habitation extending back ten thousand years. This record shows that four hundred generations lived the same lifestyle and used basically the same tools. During the course of a single lifetime, a person born in 1900 A.D. has seen the advent of radio, television, computers, automobiles, motorcycles, propeller airplanes, jet aircraft, space ships, a moon landing, test-tube babies, nuclear power generation, and nuclear weapons. Nobody can explain this sudden burst of creativity with accuracy.

In his thesis on interactive creativity, Laszlo supported his hypothesis with observations on cultural creativity (Lazlo 1994). There are documented incidences in modern sciences where different investigators have developed new scientific insight simultaneously without any known contact (Lazlo 1994). Early cultures developed tools of close resemblance; simultaneous and independent discovery of the calculus by Newton and by Leibni, biological evolution by Darwin and by Wallace are examples of creative coincidence. Similarly, Graham Bell and Elisha Grey applied the patent of the telephone on the same day. Nylon was simultaneously discovered in New York and London, hence, the name NyLon (Pandarakalam 2019).

Carl Jung researched the phenomenon of such creative synchronicity, which helped him formulate the concept of collective unconscious (Jung 1966, 1973a,b). If imagination is a property of the brain, intuition occurs in the unconscious realm. Particle physicists recognize the inspirational part is akin to the quantum leap, and the unconscious is the quantum mind (Goswami 2014). Such thoughts coming from nowhere are like mental hiccups or mind pops (Tarlaci 2019). However, the inspirational part of creativity continues to be an enigma. There may be consciousness upon consciousness. Building construction involves the participation of architects, engineers, and laborers. Similarly, creative works are the product of architects functioning in the unconscious mind, engineers working between the unconscious and the brain provides the conscious and labor force.

\section{MISDIAGNOSING CREATIVITY}

Highly cognitive children need sympathy and special attention, especially when there is "mismatching" in the family. Some children with superior intelligence mask their creative faculties by being over-talkative and overactive; these risk misdiagnosis of Attention Deficit Hyperactivity Disorder (ADHD). A creative child may figure out what the teacher is going to say in two seconds, after which he may be looking around waiting for the teacher to finish, and this would look as if the child is not paying attention. The diverted attention and the diverted achievement of creative children have to be differentiated from inattention and underachievement. The fluctuation of thoughts experienced by those with higher cognitive ability may be mistaken for mood swings (Pandarakalam 2005). An anxiety state in Einstein's mind may be diagnosed as hypomania or schizophrenia by an inexperienced psychiatrist, but his thought process was continually productive (Pandarakalam 2008). 
Creative studies are not without their limitations. The earlier investigations of 'geniuses' were retrospective. Most of the studies are confined to English-speaking people, while creativity is a global phenomenon (Pandarakalam 2019). These studies help differentiate creativity and mental illness when they coexist: the former might require nurturing, and the latter warrants clinical intervention.

\section{CONCLUSION}

This paper critically reflects on selected literature regarding creativity and its convergent versus divergent underpinnings. The authors argue that creativity is not a dichotomy between divergent and convergent thinking but, rather, a harmonious blend between the two. Based on the evidence presented within this paper, it can be said that the concept of creativity remains ambiguous, and the use of problem-solving tasks to assess this concept is limited in its construct validity. Evidence from this paper shows that both divergent and convergent thinking can function as manifestations of creative thinking. Individuals who are considered to be of high levels of creativity do not differ in performance solving task proficiency than those who are considered to be of low or moderate levels of creativity when all other variables are controlled. However, highly creative individuals do tend to utilise more divergent styles of thinking than their non-creative counterparts. This trend has led some researchers to believe that divergent thinking is inherently more creative.

We recommend further research to explore the psycho-biological nature of creativity, including genetic links, implications for neuropharmacology, and the treatment of pathology or psychological disorders. The study of creativity is valuable in developing an expanded model of the mind. The current reductionist model of mind is unhelpful in studying creativity. The assumed link between creativity and mental disorder could be clarified only when we elucidate the creative process. There is a role for an expanded brain-mind-consciousness model, which helps study the source of creativity (Pandarakalam 2021). This paper has attempted to explain the creative process from a psychological and psychiatric perspective leaving room for different unexplained aspects of generativity for open discussion.

\section{Acknowledgements: None.}

\section{Conflict of interest: None to declare.}

\section{Contribution of individual authors:}

Syed Fahad Javaid contributed to literature search, analysis and drafting of the manuscript. The two authors discussed the manuscript and approved the final version prior to submission.

James Paul Pandarakalam contributed to literature search, analysis, drafting and review of the manuscript.

\section{References}

1. Andreasen CN: Creativity and mental illness: Prevalence Rates in writers and Their First Degree Relatives. Am J Psychiatry 1987; 144:1288-1292

2. Andreasen CN \& Powers PS: Creativity and Psychosis. Arch Gen Psychiatry 1975; 32:70-73

3. Bleuler E: Dementia Praecox or the group of schizophrenias, Zinkin J (Trans). International University Press, New York, 1950

4. Braff DL \& Greyer MA: Sensorimotor gating in schizophrenia. Arch Gen Psychiatry 1990; 47:181-188

5. Carson SH, Peterson JB \& Higgins DM: Decreased latent inhibition is associated with increased creative achievement in high-functioning individuals. J Pers Soc Psychol 2003; 85:499-506

6. Carson SH: Creativity and psychopathology: a shared vulnerability model. Can J Psychiatry 2011; 56:144-153

7. Carson, SH: Cognitive Disinhibition, Creativity, and Psychopathology. Simonton DK (eds): The Wiley handbook of genius, 198-221. John Wiley \& Sons Ltd, Chichester, UK, 2014

8. Chermahini SA \& Hommel B: The link between creativity and dopamine: spontaneous eye blink rates predict and dissociate divergent and convergent thinking. Cognition 2010; 115:458-65

9. Chermahini SA \& Hommel B: Creative mood swings: divergent and convergent thinking affect mood in opposite ways. Psychol Res 2012; 76:634-40

10. Cheung PC, Lau S, Lubart T, Chu DH, Storme M: Creative potential of Chinese children in Hong Kong and French children in Paris: a cross-cultural comparison of divergent and convergent-integrative thinking. Think Skills Creat 2016; 22:201-11

11. Colzato LS, Szapora A, Lippelt D, Hommel B: Prior meditation practice modulates performance and strategy use in convergent-and divergent-thinking problems. Mindfulness 2017; 8:10-6

12. Combalt BA \& Kellp JG: Impaired attention, genetic, and the psychophysiology of schizophrenia. Schizophr Bull 1994; 20:31-46

13. Cortes RA, Weinberger AB, Daker RJ, Green AE: Re-examining prominent measures of divergent and convergent creativity. Curr Opin Behav Sci 2019; 27:90-93

14. de Vries HB \& Lubart TI: Scientific creativity: divergent and convergent thinking and the impact of culture. J Creat Behav 2019; 53:145-55

15. Donnegan KF, Setti A, Allen AP: Exercise and Creativity: Can One Bout of Yoga Improve Convergent and Divergent Thinking? J Cogn Enhanc 2018; 2:193-99

16. Duan H, Wang X, Hu W, Kounios J: Effects of acute stress on divergent and convergent problem-solving. Think Reason 2020; 26:68-86

17. Eysenck HJ: Creativity, personality, and the convergentdivergent continuum. Hampton Press, Cresskill, NJ, US, 2003

18. Gathercole CE: A note on some tests of over inclusive thinking. Br J Med Psychol 1965; 38:59-67

19. Goswami A: Quantum Creativity. Hay House, London, 2014

20. Greenwood TA. Creativity and Bipolar Disorder: A Shared Genetic Vulnerability. Annu Rev Clin Psychol 2020; 16:239-264 
21. Grohman $M G$, Ivcevic Z, Silvia P, Kaufman $S B$ : The role of passion and persistence in creativity. Psychol Aesthet Creat Arts 2017; 11:376-85

22. Jauk E, Benedek M, Neubauer AC: Tackling creativity at its roots: Evidence for different patterns of EEG alpha activity related to convergent and divergent modes of task processing. Int J Psychophysiol 2012; 84:219-25

23. Japardi K, Bookheimer S, Knudsen K, Ghahremani DG, Bilder RM: Functional magnetic resonance imaging of divergent and convergent thinking in Big-C creativity. Neuropsychologia 2018; 118:59-67

24. Jung C: The Psychology of the unconscious, in The Collected Works of C.G. Jung. Vol. vii, Princeton university Press, Princeton, NJ, US, 1966

25. Jung C: Psychology and Literature. In The Creative Process, Ghiseline B, Ed. The New American Library, New York, 1973 a

26. Jung C: Synchronicity, a casual connecting principle. Collective Works. Vol.viii, Princeton University Press, Princeton, NJ, US, $1973 b$

27. Laszlo E: The Genius Hypothesis: Exploratory concepts for a scientific understanding of unusual creativity. $J$ Sci Explor 1994; 18:257-267

28. Lu JG, Akinola M, Mason MF: Switching On creativity: Task switching can increase creativity by reducing cognitive fixation. Organ Behav Hum Decis Process 2017; 139:63-75

29. McCrae RR: Creativity, divergent thinking, and openness to experience. J Pers Soc Psychol 1987; 52:1258-1265

30. McDonald H, Babunashvili M, Finn A, Willard A, Valmaggia L, Chadwick $P$ et al.: Positive schizotypy and the experience of creativity: The distinctive roles of suspiciousness and dispositional mindfulness. Schizophr Res 2021; 228:151-158

31. Mekern V, Hommel B, Sjoerds Z: Computational models of creativity: a review of single-process and multi-process recent approaches to demystify creative cognition. Curr Opin Behav Sci 2019; 27:47-54

32. O'Brian JP \& Weingartner H: Associative structure in schizophrenia. Arch Gen Psychiatry 1970; 22:136-148

33. Pandarakalam JP: Psychopathological views of creativity: an analysis. The Irish Psychiatrist 2005; 6:117-121

34. Pandarakalam JP: Diagnostic Conundrums of Bipolar Disorder. Prog Neurol Psychiatry 2008; 12:6-11

35. Pandarakalam JP: Conundrums of the Association between Creativity and Psychopathology. Br J Med Pract 2019; 12:a022

36. Pandarakalam JP: Contribution of Cases of Extraordinary Healings to the Study of Consciousness. Neuro Quantology 2021; 19:1-12
37. Preti A: The role of dopaminergic pathway in the neurobiology of schizophrenia. Critical issues in the treatment of schizophrenia 1995. Florence: 102-103

38. Razoumnikova O: Function Organization of Different Brain Areas During Convergent and Divergent Thinking: an EEG Investigation. Cogn Brain Res 2000; 10:11-18

39. Rominger C, Fink A, Weiss EM, Bosch J, Papousek I: Allusive thinking (remote associations) and auditory top-down inhibition skills differentially predict creativity and positive schizotypy. Cogn Neuropsychiatry 2017; 22:108-121

40. Rothenberg A: The process of Janusian thinking in creativity. Arch Gen Psychiatry 1971; 24:195-205

41. Rothenberg A: Opposite responding as a measure of creativity. Psychol Rep 1973; 33:15-18

42. Rothenberg A: Einstein's creative thinking and the general theory of relativity: A documented report. Am J Psychiatry 1979; 136:38-43

43. Rothenberg A: Janusian Thinking and Nobel Prize Laureates. Am J Psychiatry 1982; 139:122-124

44. Rybakowski JK \& Klonowska P. Bipolar mood disorder, creativity and schizotypy: an experimental study. Psychopathology 2011; 44:296-302

45. Said-Metwaly S, Van den Noortgate W, Kyndt E: Approaches to measuring creativity: a systematic literature review. Creativity. Theories-Research-Applications 2017; 4:238-75

46. Schuldberg D: Schizotypal and hypomanic traits, creativity, and psychological health. Creat Res J 1990; 3:557-56

47. Shen W, Hommel B, Yuan Y, Chang L, Zhang W: Risktaking and creativity: convergent, but not divergent thinking is better in low-risk takers. Creat Res J 2018; 30:224-31

48. Schwartz S: Language and cognition in schizophrenia. Lawrence Erlbaum, Hillside, NJ, 1978

49. Tarlaci S: Neuroquantology, Quantum Physics in the Brain. Nova Science Publishers, New York, 2014

50. Tarlaci S: Quantum neurobiological view to mental health problems and biological psychiatry. Journal of Psychopathology 2019; 25:70-84

51. Wronska MK, Bujacz A, Gocłowska MA, Rietzschel EF, Nijstad BA: Person-task fit: Emotional consequences of performing divergent versus convergent thinking tasks depend on need for cognitive closure. Pers Individ Differ 2019; 142:172-8

52. Yang KK, Hong ZR, Lee L, Lin HS: Exploring the significant predictors of convergent and divergent scientific creativities. Think Skills Creat 2019; 31:252-61

53. Zhu W, Shang S, Jiang W, Pei M, Su Y: Convergent Thinking Moderates the Relationship between Divergent Thinking and Scientific Creativity. Creat Res J 2019; 31:320-8

Correspondence:

Assistant Professor Syed Fahad Javaid, MD, MBBS MSc MRCPsych

Department of Psychiatry and Behavioral Sciences, College of Medicine and Health Sciences,

United Arab Emirates University

PO Box 17666, Al Ain, Abu Dhabi, United Arab Emirates

E-mail:sjavaid@uaeu.ac.ae 